\title{
RADICALS OF 0-REGULAR ALGEBRAS
}

\author{
N.R. McCONNELL and TIM STOKES
}

\begin{abstract}
We consider a generalisation of the Kurosh-Amitsur radical theory for rings (and more generally multi-operator groups) which applies to 0-regular varieties in which all operations preserve 0 . We obtain results for subvarieties, quasivarieties and element-wise equationally defined classes. A number of examples of radical and semisimple classes in particular varieties are given, including hoops, loops and similar structures. In the first section, we introduce 0 -normal varieties (0-regular varieties in which all operations preserve 0 ), and show that a key isomorphism theorem holds in a 0-normal variety if it is subtractive, a property more general than congruence permutability. We then define our notion of a radical class in the second section. A number of basic results and characterisations of radical and semisimple classes are then obtained, largely based on the more general categorical framework of L. Márki, R. Mlitz and R. Wiegandt as in [13]. We consider the subtractive case separately. In the third section, we obtain results concerning subvarieties and quasivarieties based on the results of the previous section, and also generalise to subtractive varieties some results for multi-operator group radicals defined by simple equational rules. Several examples of radical and semisimple classes are given for a range of fairly natural 0-normal varieties of algebras, most of which are subtractive.
\end{abstract}

12

\section{Generalities}

We are interested in 0-regular varieties in which $\{0\}$ is always a subalgebra; this includes of course all varieties of multi-operator groups, but also loops, hoops and various other fairly natural examples, which we return to later. 0-regularity of a variety can be described in terms of a so-called Malcev condition: that there exist finitely many binary terms $d_{i}(x, y)$ such that $d_{i}(x, x)=0$ for all $i$, and if $d_{i}(x, y)=0$ for all $i$ then $x=y$.

Thus let $\mathcal{V}$ be a variety of algebras with nullary operation the distinguished zero element 0 . If for each congruence $\rho$ on each $A \in \mathcal{V}$, the $\rho$-class containing 0 determines $\rho$ then $\mathcal{V}$ is a 0 -regular variety. If also $\sigma(0,0, \ldots, 0)=0$ is an identity for each operation $\sigma$ in the signature of $\mathcal{V}$, then we call $\mathcal{V}$ a 0 -normal variety.

\footnotetext{
${ }^{1}$ Key words and phrases: radical class, 0-regular variety, subtractive variety.

2 2000 Mathematics Subject Classification: primary 08C99; secondary 08A99, 08B99.
} 
Let $\mathcal{V}$ be a 0 -normal variety. We shall call those subsets of algebras in $\mathcal{V}$ which are the congruence classes containing 0 normals; they are the kernels of homomorphisms in the obvious sense, and are subalgebras. The normals of an algebra evidently form a lattice in which meet is intersection, isomorphic to the lattice of congruences. We write $N \triangleleft A$ if $N$ is a normal in $A \in \mathcal{V}$, and we denote by 0 the normal $\{0\}$ in any $A$. For $N \triangleleft A$, let $\rho_{N}$ denote the corresponding congruence, and for $\rho$ a congruence on $A$ let $N_{\rho}$ denote the corresponding normal. Then for any $N \triangleleft A$ and congruence $\rho$ on $A, \rho_{N_{\rho}}=\rho$ and $N_{\rho_{N}}=N$; we write $A / N$ rather than $A / \rho_{N}$, in keeping with the tradition for groups, rings and so on.

The usual facts of universal algebra hold in 0-normal varieties, and some of those from group and ring theory. Thus, if $f: A \rightarrow B$ is a homomorphism between algebras in a 0-normal variety, then $\operatorname{ker}(f)=\{a \in A \mid f(a)=0\} \triangleleft A$. Moreover, $A / \operatorname{ker}(f) \cong \operatorname{Im}(f)$. It follows easily that if $N \triangleleft A$ and $B$ is a subalgebra of $A$ with $N \subseteq B$, then $N \triangleleft B$. Generally, we shall deal with universal classes within 0-normal varieties, that is, classes closed under taking normals and homomorphic images.

Let $\mathcal{V}$ be a variety. If all congruences on any $A \in \mathcal{V}$ permute, then $\mathcal{V}$ is a Malcev variety. Malcev's well-known theorem says that a variety is Malcev if and only if there is a ternary term $w(x, y, z)$ for which $w(x, x, y)=y$ and $w(x, y, y)=x$. A less well-known result, indirectly shown in [10], and later demonstrated explicitly in [1] (see Theorem 2.4), concerns varieties $\mathcal{V}$ with distinguished nullary 0 . Two congruences $\rho, \theta$ on $A$ permute at zero if $(a, 0) \in \rho \circ \theta$ implies $(a, 0) \in \theta \circ \rho$ and vice versa; equivalently, $(a, 0) \in \rho \vee \theta$ implies the existence of $b \in A$ for which $(b, 0) \in \rho$ and $(a, b) \in \theta$. The result then says that all algebras in a variety have congruences permuting at zero if and only if there is a binary term $s(x, y)$ in the variety for which $s(x, x)=0$ and $s(x, 0)=x$. Such varieties are called subtractive in [18].

Any variety of multi-operator groups is of course a subtractive variety; normals are normal subgroups in varieties of groups, ideals in varieties of rings, submodules in varieties of modules, and so on. Other examples of subtractive varieties include loops and hoops, among others which we return to later. Note that in the current 0-normal setting, subtractive varieties are exactly ideal determined varieties, in the sense of [10].

The following statement and its corollary may be known to people working with these notions but we have not seen them in print and therefore present them for the sake of completeness.

PROPOSITION 1 For $A \in \mathcal{V}$, a 0-normal variety, if $M, N \triangleleft A$ and $\rho_{M}, \rho_{N}$ permute at zero, then $M /(M \cap N) \cong(M \vee N) / N$.

PROOF. First note that both sides of the isomorphism are well-defined. Define $f: M \rightarrow(M \vee$ $N) / N$ by setting $f(a)=a N$, where $a N$ is the congruence class containing $a$ in $(M \vee N) / N$. This is easily seen to be a homomorphism with kernel $M \cap N$. It remains to prove surjectivity. We must show that for any $b \in M \vee N$, there is $a \in M$ such that $(a, b) \in \rho_{N}$; that is, for any $(b, 0) \in \rho_{M \vee N}=\rho_{M} \vee \rho_{N}$, there is $(a, 0) \in \rho_{M}$ such that $(a, b) \in \rho_{N}$. This is immediate from the 
fact that $\rho_{M}, \rho_{N}$ permute at zero.

Note that the homomorphism $f$ in the above proof exists whether or not $\rho_{M}$ and $\rho_{N}$ permute at zero, but is surjective if and only if they do. It therefore seems likely that permutability at zero is equivalent to the above isomorphism holding, if not for specific pairs of normals $M, N$ then for whole algebras, or perhaps at least for varieties. However, each of these conjectures is open at present. In any case, we have the following immediate corollary.

COROLLARY 1 In a subtractive variety $\mathcal{V}$, the isomorphism theorem $M /(M \cap N) \cong(M \vee N) / N$ holds for normals $M, N$ of any $A \in \mathcal{V}$.

We generally deal with universal classes in what follows. We shall say that any universal class in a subtractive variety is a subtractive universal class.

\section{Radical and semisimple classes}

Throughout this section, let $\mathcal{A}$ be a universal class contained in some 0 -normal variety of algebras.

\section{$2.1 \quad$ Radical classes}

The definition to follow is in part by analogy with the usual definition for rings and multi-operator groups, but also in part inspired by the definition of a radical class of idempotent algebras appearing in [9]. We say a subclass $\mathcal{R}$ of $\mathcal{A}$ is a radical class if the following conditions hold.

- (R1) If $I, J \triangleleft A$ with $I \in \mathcal{R}$, then there exists $K \triangleleft A / J$ for which $(I \vee J) / J \subseteq K \in \mathcal{R}$.

- (R2) For each $A \in \mathcal{A}$, there exists $\mathcal{R}(A) \triangleleft A$ (the radical of $A$ ) such that $\mathcal{R}(A) \in \mathcal{R}$ and for $J \triangleleft A$ such that $J \in \mathcal{R}, J \subseteq \mathcal{R}(A)$.

- $(\mathrm{R} 3) \mathcal{R}(A / \mathcal{R}(A))=0$ for any $A$.

Note the strengthening of the first axiom compared to the usual homomorphic closure:

- (H) $I \triangleleft A$ and $A \in \mathcal{R}$ imply $A / I \in \mathcal{R}$.

It is easy to see that a class satisfying (R1) must satisfy $(\mathrm{H})$ also: simply let $I=A$ and then necessarily $K=A / J$. (R1) is in fact equivalent to $(\mathrm{H})$ in the subtractive universal class setting as we show shortly, but (R1) seems to be needed in the more general case in order to prove results of any strength. Still, we so far have no examples to show the simpler axiom $(\mathrm{H})$ is insufficient. (In the idempotent algebras case discussed in [9], examples are given).

Our definition is in fact a special case of a much more general categorical definition given by Márki, Mlitz and Wiegandt in [13]. Not wishing to spend time on the detail here, the reader is invited to consult this paper where appropriate. Proposition 3.4 in [13] establishes that the radical classes considered here are approximable, and all consequences of this fact then follow immediately. 
If $\mathcal{R}$ is a radical class, $A \in \mathcal{A}$ is said to be $\mathcal{R}$-radical if $\mathcal{R}(A)=A$ (equivalently, if $A \in \mathcal{R}$ ) and $\mathcal{R}$-semisimple if $\mathcal{R}(A)=0$. An ideal $I$ of $A$ is an $\mathcal{R}$-ideal if $I \in \mathcal{R}$.

PROPOSITION 2 The class $\mathcal{R}$ is a radical class if and only if it satisfies (R2), (R3), and for every $a \in \mathcal{A}$ and $I \triangleleft A,(\mathcal{R}(A) \vee I) / I \subseteq \mathcal{R}(A / I)$, where $\mathcal{R}(A)$ is as for (R2) above.

PROOF. Suppose $\mathcal{R}$ is a radical class. If $I \triangleleft A$ then by (R1) there exists $K \triangleleft A / I$ with $K \in \mathcal{R}$ for which $(\mathcal{R}(A) \vee I) / I \subseteq K$. Since $K \subseteq \mathcal{R}(A / I)$ by (R2), the result follows.

Conversely, suppose $\mathcal{R}$ is a class for which (R2) and (R3) hold, and that for every $A \in \mathcal{A}$ and $I \triangleleft A,(\mathcal{R}(A) \vee I) / I \subseteq \mathcal{R}(A / I)$. Then if $I, J \triangleleft A$ with $I \in \mathcal{R}$, let $K=\mathcal{R}(A / J)$. Then $(I \vee J) / J \subseteq \mathcal{R}(A / J)=K \in \mathcal{R}$.

In a 0 -normal variety, this proposition shows that $(\mathrm{R} 1)$ is needed to ensure radicals are radical in the sense of Hoehnke, [11]: if $\rho_{A}$ is the induced congruence in $A \in \mathcal{U}$ associated with $\mathcal{R}$ as in (R2), the property in the proposition says that $f\left(\rho_{A}\right) \subseteq \rho_{B}$ for all surjective homomorphisms $f: A \rightarrow B$.

Aside from (H) above, some further properties that a class $\mathcal{R}$ in $\mathcal{A}$ may or may not satisfy are:

- (E) If $I \triangleleft A$ and both $I$ and $A / I$ are in $\mathcal{R}$, then $A \in \mathcal{R}$.

- (C) If $\left\{I_{\theta} \mid \theta \in \Omega\right\}$ is a chain of ideals of $a$ such that $I_{\theta} \in \mathcal{R}$ for all $\theta$, then $\bigvee I_{\theta} \in \mathcal{R}$.

THEOREM $1 \mathcal{R}$ is a radical class if and only if it satisfies (R1), (E) and (C).

PROOF. This follows from results in [13]: in particular regarding p.284, the M-systems in the current case (basically normals) are inductive, and trivially satisfy the "special congruence extension property". The characterization given there is precisely the one wanted here (when easily translated).

PROPOSITION 3 Suppose $\mathcal{A}$ is subtractive and $\mathcal{X}$ is a class in $\mathcal{A}$. Then $\mathcal{X}$ satisfies (R1) if and only if $\mathcal{X}$ satisfies $(H)$.

PROOF. Suppose $\mathcal{X}$ satisfies $(\mathrm{H})$, with $I, J \triangleleft A$ for which $I \in \mathcal{X}$. Then by assumption, $K=$ $(I \vee J) / J \cong I /(I \cap J) \in \mathcal{X}$ by $(\mathrm{H})$, and (R1) is clearly satisfied.

Hence in a subtractive universal class, a class is a radical class if and only if it satisfies $(\mathrm{H}),(\mathrm{E})$ and $(\mathrm{C})$, and we recover the familiar definition for associative rings. Note also that in this case we have radical classes in the sense of [16]; hence all Puczyłowski's results apply (though many of these are also special cases of results in [13]). Note also that the subtractive case corresponds to assuming the "M-relation" (in the sense of [13]) is homomorphically closed, so all relevant results apply. 


\section{$2.2 \quad$ Semisimple classes}

As usual in general algebra, we say $A$ is a subdirect product of $A / I_{\lambda}$, where the $I_{\lambda}, \lambda \in \Lambda$ are normals of $A$ for which $\bigwedge I_{\lambda}=0$. A class $\mathcal{X}$ in $\mathcal{A}$ is closed under subdirect products if the existence of normals $I_{\lambda}, \lambda \in \Lambda$ of $A \in \mathcal{A}$ for which $\bigwedge I_{\lambda}=0$ and each $A / I_{\lambda} \in \mathcal{X}$ implies that $A \in \mathcal{X}$ also.

$\mathcal{S}$ is a semisimple class in $\mathcal{A}$ if there is a radical class $\mathcal{R}$ such that $A \in \mathcal{S}$ if and only if $\mathcal{R}(A)=0$. Hence by Theorem 2.6 of [13], semisimple classes can be characterised by the definition given on p.265 of that paper, and they are subdirectly closed by Proposition 2.3.

For a class $\mathcal{X}$ containing 0 in $\mathcal{V}$ and for any $A \in \mathcal{V}$, associate the ideal $A(\mathcal{X})=\bigwedge\{I \triangleleft A \mid A / I \in$ $\mathcal{X}\}$.

THEOREM $2 \mathcal{S}$ is a semisimple class if and only if

- $(S 0) 0 \in \mathcal{S}$;

- (S1) $I \triangleleft A$ and $A / I \in \mathcal{S}$ imply that for all $J \triangleleft A$ for which $J \nsubseteq I, J$ has a proper ideal $K$ such that $J / K \in \mathcal{S}$;

- (S2) $\mathcal{S}$ is closed under subdirect products; and

- (S3) $A(\mathcal{S})(\mathcal{S})=A(\mathcal{S})$ for every $A$.

PROOF. Suppose $\mathcal{R}$ is a radical class, with $\mathcal{S}=\{A \mid \mathcal{R}(A)=0\}$. That (S2) holds follows from Proposition 2.3 of [13].

For any $A, A / \mathcal{R}(A) \in \mathcal{S}$, so $A(\mathcal{S}) \subseteq \mathcal{R}(A)$. Because it is a subdirect product of $A / I$ 's which are in $\mathcal{S}, A / A(\mathcal{S}) \in \mathcal{S}$ by $(\mathrm{S} 2)$. So by Proposition $2,(\mathcal{R}(A) \vee A(\mathcal{S})) / A(\mathcal{S}) \subseteq \mathcal{R}(A / A(\mathcal{S}))=0$, so $\mathcal{R}(A)=A(\mathcal{S})$.

Proposition 2.10 of [13] immediately gives us that $\mathcal{S}$ satisfies (S1).

Finally, $A(\mathcal{S})(\mathcal{S})=\mathcal{R}(\mathcal{R}(A))=\mathcal{R}(A)=A(\mathcal{S})$. So (S3) holds.

To the converse, suppose the non-empty class $\mathcal{S}$ satisfies (S0) to (S3). Let $\mathcal{R}=\{A \mid A / I \in$ $\mathcal{S}$ implies $I=A\}=\{A \mid A(\mathcal{S})=A\}$. By $(\mathrm{S} 3), A(\mathcal{S}) \in \mathcal{R}$ for all $A$, and $\bar{A}=A / A(\mathcal{S}) \in \mathcal{S}$ by (S2), so $\mathcal{S}(\bar{A})=0$. So $(A / A(\mathcal{S}))(\mathcal{S})=0$.

Let $I \triangleleft A, I \in \mathcal{R}$. So $I$ has no non-zero factor in $\mathcal{S}$. By (S1), $I \subseteq J$ for each $J \triangleleft A$ such that $A / J \in \mathcal{S}$. So $I \subseteq \bigcap\{J \mid A / J \in \mathcal{S}\}=A(\mathcal{S})$. So setting $\mathcal{R}(A)=A(\mathcal{S})$, (R2) is clearly satisfied. But so is $(\mathrm{R} 3)$ since $(A / A(\mathcal{S}))(\mathcal{S})=0$ as just shown.

Finally, if $J \triangleleft A$ with $A / J \in \mathcal{S}$, then $J \supseteq A(\mathcal{S})$, and if $J \supseteq I \triangleleft A$, then $J \supseteq A(\mathcal{S}) \vee I$. Hence $(A(\mathcal{S}) \vee I) / I \subseteq J / I \triangleleft A / I$, with $J / I$ a typical ideal of $A / I$ for which $(A / I) /(J / I) \cong A / J \in \mathcal{S}$. Hence $(A(\mathcal{S}) \vee I) / I \subseteq(A / I)(\mathcal{S})$, so (R1) is satisfied. Thus $\mathcal{R}$ is a radical class, with $\mathcal{R}(A)=A(\mathcal{S})$. The semisimple class corresponding to $\mathcal{R}$ is $\{A \mid \mathcal{R}(A)=0\}=\{A \mid A(\mathcal{S})=0\}$. But this is $\mathcal{S}$; for if $A \in \mathcal{S}$ then obviously $A(\mathcal{S})=\bigcap\{I \mid A / I \in \mathcal{S}\}=0$, and if $A(\mathcal{S})=\bigcap\{I \mid A / I \in \mathcal{S}\}=0$ then $A$ is a subdirect product of the $A / I$ 's in $\mathcal{S}$ and hence is itself in $\mathcal{S}$ by (S2). 
There is an inclusion-reversing bijection between the collections of radical and semisimple classes in $\mathcal{U}$, as follows from Theorem 2.6 of [13] essentially.

THEOREM $3 \mathcal{S}$ is a semisimple class in the subtractive universal class $\mathcal{A}$ if and only if (S2),

(S3) and

$\left(S 1^{\prime}\right) a \in \mathcal{S}$ implies that every non-zero $I \triangleleft A$ is such that there is a proper ideal $J \triangleleft I$ such that $I / J \in \mathcal{S}$.

PROOF. We must show (S1') implies (S1). Suppose $A / I \in \mathcal{S}$, with $J \triangleleft A$ such that $J \nsubseteq I$. Then $I \nsubseteq J \vee I$ and $(J \vee I) / I \neq 0$ so $J /(J \cap I) \cong(J \vee I) / I \triangleleft A / I$ has a proper ideal $K$ such that $(J /(J \cap I)) / K \in \mathcal{S}$ by $\left(\mathrm{S} 1^{\prime}\right)$. It now follows easily that $J$ itself has a proper ideal $M$ with $J / M \in \mathcal{S}$.

The condition (S3) is necessary in general: although it can be replaced by (E) in the case of associative rings, for non-associative rings it cannot.

\section{Classes defined by equations and implications}

In this section we consider further results and examples involving varieties and quasivarieties as well as element-wise defined classes.

\subsection{Varieties}

Let $\mathcal{V}$ be a variety in the universal class $\mathcal{U}$ of 0 -normal algebras (itself contained in some 0 -normal variety). Letting $A(\mathcal{V})=\bigcap\{I \mid A / I \in \mathcal{V}\}$, as before, it is clear that $A / A(\mathcal{V})$ is a subdirect product of algebras in $\mathcal{V}$ and hence is itself in $\mathcal{V}$. We say $\mathcal{V}$ has attainable identities if $A(\mathcal{V})(\mathcal{V})=A(\mathcal{V})$ for all $A \in \mathcal{V}$, that is, if $\mathcal{V}$ satisfies (S3) in the characterisation of semisimple classes given in Theorem 2. It is easy to see that if $\mathcal{V}$ has attainable identities then it satisfies (E), since if $I \triangleleft A$ and $I, A / I \in \mathcal{V}$, then $A(\mathcal{V}) \subseteq I$, so $A(\mathcal{V}) \in \mathcal{V}$ and so $A(\mathcal{V})=A(\mathcal{V})(\mathcal{V})=0$, and so $A \in \mathcal{V}$.

PROPOSITION 4 A variety $\mathcal{W}$ in the universal class $\mathcal{U}$ is a radical class if and only if it satisfies (R1) and (E).

PROOF. Let $\mathcal{W}$ be a variety. Let $\left\{I_{\sigma} \mid \sigma \in \Lambda\right\}$ be a chain of $\mathcal{W}$-ideals in some $A$. Then the directed join $I=\bigvee\left\{I_{\sigma} \mid \sigma \in \Lambda\right\} \in \mathcal{V}$ since varieties are closed under directed unions, so (C) is satisfied. The result now follows from Proposition 1.

Because radical classes are characterised by $(\mathrm{H}),(\mathrm{E})$ and $(\mathrm{C})$ in the subtractive case, we obtain the following corollary to this proof.

COROLLARY 2 A variety $\mathcal{V}$ in the subtractive universal class $\mathcal{U}$ is a radical class if and only if it satisfies (E). 
PROPOSITION 5 A semisimple class satisfying $(H)$ is a variety.

PROOF. Suppose $\mathcal{S}$ is semisimple and satisfies $(\mathrm{H})$. Then by Theorem 2, it is closed under subdirect products, so $\mathcal{S}$ is a variety by Kogalovskii's Theorem [12]; see also Theorem IV.3.5 (attributed to Hall) in [5].

LEMMA 1 (S1) holds in any variety.

PROOF. Let $\mathcal{V}$ be a variety in $\mathcal{U}$. Let $I \triangleleft A$ have no proper ideal $J$ for which $I / J \in \mathcal{V}$, that is, no non-zero homomorphic image in $\mathcal{V}$. Then the canonical homomorphism $\psi: A \rightarrow A / A(\mathcal{V})$ takes $I$ to $S$, which, though not necessarily a normal, is a subalgebra of $A / A(\mathcal{V})$ since $I$ is a subalgebra of $A$. Since $A / A(\mathcal{V}) \in \mathcal{V}$, so is $S$. However, $S$ is a homomorphic image of $I$ in $\mathcal{V}$ which by assumption must be 0 . Hence $I \subseteq A(\mathcal{V})$. But if $K \triangleleft A$ with $A / K \in \mathcal{V}$, then $A(\mathcal{V}) \subseteq K$ of course, so $I \subseteq K$, and $(\mathrm{S} 1)$ is shown.

Note that an analogous result appears to hold for idempotent algebras as in [9]: attainability of identities is not needed there either, although the author's proof seems to make use of it.

THEOREM 4 A variety $\mathcal{V}$ is a semisimple class if and only if it has attainable identities.

PROOF. Suppose the variety $\mathcal{V}$ has attainable identities. By the previous result it satisfies (S1), and (S0) and (S2) are immediate. The converse direction is immediate from Theorem 2.

A semisimple radical class, or SSR class, is a class which is both a semisimple and a radical class.

THEOREM 5 A semisimple class is an SSR class if and only if it satisfies (R1).

PROOF. Suppose $\mathcal{S}$ is semisimple and satisfies (R1). By Proposition $5, \mathcal{S}$ is a variety and so it has attainable identities by Theorem 4. Now if $A / I$ and $I$ are in $\mathcal{S}, I \triangleleft A$, then $A(\mathcal{S}) \subseteq I$, so $A(\mathcal{S}) \in \mathcal{V}$ also, and so $A(\mathcal{S})(\mathcal{S})=0$, so $A(\mathcal{S})=0$ by attainability. But $A / A(\mathcal{S}) \in \mathcal{S}$ since $A / A(\mathcal{S})$ is a subdirect product of things in $\mathcal{S}$, a semisimple class. Hence $A \cong A / 0 \in \mathcal{S}$ and so satisfies (E). Thus $\mathcal{S}$ is a radical class by Proposition 4 . The converse is immediate.

THEOREM 6 Let $\mathcal{U}$ be a subtractive universal class.

1. $\mathcal{S}$ is an SSR class if and only if it is a subvariety with attainable identities.

2. A semisimple class $\mathcal{S}$ is an SSR class if and only if it satisfies (H).

PROOF.

1. By Proposition 5 any SSR class is a variety, and by Theorem 2 any semisimple class has attainable identities. Conversely, a variety with attainable identities is semisimple by Theorem 4 , and satisfies (E) and hence is radical by Corollary 2. 
2. If $\mathcal{S}$ is semisimple and satisfies $(\mathrm{H})$, then by Proposition 5 it is a variety and so satisfies (S3) by Theorem 4, that is, it has attainable identities, and thus satisfies (E); hence it is a radical class by Corollary 2 .

As we have seen, in any variety of 0-normal algebras, the implication "attainable identities implies (E)" holds. In many subtractive varieties, the reverse implication holds for subvarieties, for instance in associative rings. The following sufficient conditions can be useful, and generalise results cited in [7]. (The proof of the theorem is very similar but we include it for completeness.)

THEOREM 7 Let $\mathcal{V}$ be a variety in the subtractive universal class $\mathcal{U}$, with $\mathcal{V}$ satisfying $(E)$. Then for every $A \in \mathcal{U}, A(\mathcal{V})$ is the normal of $A$ generated by $A(\mathcal{V})(\mathcal{V})$.

PROOF. Let $I$ be the normal generated by $A(\mathcal{V})(\mathcal{V})$. Now $A /(A(\mathcal{V}) \vee I) \in \mathcal{V}$ since it is a homomorphic image of $A / A(\mathcal{V}) \in \mathcal{V}$. Thus $\mathcal{V} \ni A /(A(\mathcal{V}) \vee I) \cong(A / I) /((A(\mathcal{V}) \vee I) / I)$ with $(A(\mathcal{V}) \vee I) / I \cong A(\mathcal{V}) /(A(\mathcal{V}) \cap I)$. But $A(\mathcal{V})(\mathcal{V})$ is a subset of both $A(\mathcal{V})$ and $I$, so $A(\mathcal{V}) /(A(\mathcal{V}) \cap I)$ is a homomorphic image of $A(\mathcal{V}) / A(\mathcal{V})(\mathcal{V})$ and hence is in $\mathcal{V}$. It follows from (E) that $A / I \in \mathcal{V}$, so $A(\mathcal{V}) \subseteq I$. But $A(\mathcal{V})(\mathcal{V}) \triangleleft A(\mathcal{V}) \triangleleft A$, so $I \subseteq A(\mathcal{V})$ also, and the result follows.

COROLLARY 3 If normals are transitive in the subtractive universal class $\mathcal{U}$, then any variety in $\mathcal{U}$ satisfying $(E)$ has attainable identities and hence is an SSR class.

Such varieties are therefore radical if and only if they are semisimple, if and only if (E) holds.

\subsection{Quasivarieties}

Let $\mathcal{V}$ be a 0 -normal variety throughout this section, with $\mathcal{U}$ a contained universal class, and let $F r_{\Omega}$ be the free algebra in $\mathcal{V}$ on the generators $x_{1}, x_{2}, \ldots$ For $f \in F r_{\Omega}$, we write $f\left(x_{i}\right)$ as shorthand for $f\left(x_{1}, x_{2}, \ldots, x_{k}\right)$ (where $k$ is the largest index of any variable occurring in $f$ ), and similarly $f\left(a_{i}\right)$ is the result of evaluating $f$ with $x_{i}$ replaced by $a_{i} \in A, A \in \mathcal{V}$.

Recall that a quasivariety in $\mathcal{U}$ is a subclass defined by implications of the form

$$
f_{1}=g_{1} \& f_{2}=g_{2} \& \ldots \& f_{k}=g_{k} \Rightarrow f=g
$$

where the $f_{i}=g_{i}$ and $f=g$ are equations in $\mathcal{V}$, so that all $f_{i}, g_{i}$ as well as $f, g$ are in $F r_{\Omega}$. Varieties are quasivarieties: $f=g$ is equivalent to $x=x \Rightarrow f=g$.

Quasivarieties are closed under subalgebras, direct products (and filtered products), so to be radical classes, they must be varieties; more interesting then are semisimple classes.

Suppose $\mathcal{W}$ is a quasivariety in $\mathcal{U}$. Then as for varieties, letting $A(\mathcal{W})=\bigcap\{I \mid A / I \in \mathcal{W}\}$, $A / A(\mathcal{W})$ is itself in $\mathcal{W}$. Again, if $\mathcal{W}$ satisfies $(\mathrm{S} 3)$ in Theorem 2, that is if $A(\mathcal{W})(\mathcal{W})=A(\mathcal{W})$ for all $A \in \mathcal{V}$, then $\mathcal{W}$ satisfies (E).

Because Lemma 1 holds for quasivarieties (the proof is identical), the relevant version of Theorem 4 follows easily too. 
THEOREM 8 A quasivariety $\mathcal{W}$ is a semisimple class if and only if (S3) in the characterisation of a semisimple class holds: $A(\mathcal{W})(\mathcal{W})=A(\mathcal{W})$ for all $A \in \mathcal{U}$.

In many universal classes, (E) is sufficient in place of (S3), as for varieties; for instance associative and alternative rings, as well as groups and modules. The following result is shown for rings and groups in [7], although the proof given there goes across to any universal class of algebras in a 0 -normal variety without modification.

PROPOSITION 6 Any quasivariety with defining implications of the following form satisfies $(E)$ :

$$
f\left(x_{i}\right)=g\left(x_{i}\right) \Rightarrow x_{i}=0 \text { for all } i .
$$

It turns out that having transitive normals is sufficient for any such quasivariety to satisfy (S3) and hence be a semisimple class. This provides a rich source of examples of semisimple classes, even in the non-subtractive case (see the final section).

THEOREM 9 In a universal class in which normals are transitive, any quasivariety $\mathcal{W}$ with defining implications as in Proposition 6 satisfies $(S 3), A(\mathcal{W})(\mathcal{W})=A(\mathcal{W})$ for all $A \in \mathcal{U}$, and hence is a semisimple class.

PROOF. If $\mathcal{W}$ is such a quasivariety, suppose $A \in \mathcal{U}$. Then clearly

$$
A(\mathcal{W})=\cap\left\{I \mid I \triangleleft A, f\left(a_{i}\right) \rho_{I} g\left(a_{i}\right) \text { implies all } a_{i} \in I\right\}
$$

Now by transitivity, $A(\mathcal{W})(\mathcal{W}) \triangleleft A$. Suppose $a_{i} \in A$ are such that $f\left(a_{i}\right) \rho_{A(\mathcal{W})(\mathcal{W})} g\left(a_{i}\right)$. Then certainly $f\left(a_{i}\right) \rho_{A(\mathcal{W})} g\left(a_{i}\right)$, since $A(\mathcal{W})(\mathcal{W}) \subseteq A(\mathcal{W})$, so all $a_{i} \in A(\mathcal{W})$ with $f\left(a_{i}\right) \rho_{A(\mathcal{W})(\mathcal{W})} g\left(a_{i}\right)$, so also each $a_{i} \in A(\mathcal{W})(\mathcal{W})$. Hence $A(\mathcal{W})(\mathcal{W})$ is a normal $I$ of $A$ for which $f\left(a_{i}\right) \rho_{I} g\left(a_{i}\right)$ implies all $a_{i} \in I$, and so contains $A(\mathcal{W})$; hence they are equal.

\subsection{Element-wise equationally defined radical classes}

Another fertile source of radical classes, similar in spirit to the previous two, is via element-wise equational definitions of the form "for all $x \in A$ there is $y \in A$ for which $f(x, y)=0$ ". The idea generalises readily to more than one $f$ and more than one $y$ (or even none). In the subtractive case, such a class is guaranteed to be radical by a relatively simple condition on the terms used to define it. The Jacobson, regular and nil radicals of ring theory are then special cases, as are many others. See [14] for the multi-operator group special case of this concept.

Again let $\mathcal{V}$ be a 0 -normal variety throughout this section, with $\mathcal{U}$ a contained universal class. Here let $F r_{\Omega}$ be the free algebra in $\mathcal{V}$ on the generators $x, y_{1}, y_{2}, \ldots$. For any $F \subseteq F r_{\Omega}$, let $\mathcal{R}_{F}$ be the class of algebras in $\mathcal{V}$, defined as follows: $R$ is in $\mathcal{R}_{F}$ providing that, for every $r \in R$ there exist $f \in F$ and $s_{i} \in R$ such that $f\left(r, s_{i}\right)=0$.

If $\mathcal{V}$ is the variety of associative rings, the classes of quasiregular and von Neumann regular rings have the form $\mathcal{R}_{\{f\}}$ for some $f \in \Omega$ : in the former case, we may let $f=x+y+x y$; in the 
latter, $f=x-x y x$. Similarly the class of nil rings is $\mathcal{R}_{F}$ where $F=\left\{x^{n} \mid n>0\right\}$. This idea can be generalised to give a method for generating radical classes in subtractive varieties. Again, in the subtractive case, the characterisation of radical classes as satisfying $(\mathrm{H}),(\mathrm{E})$ and $(\mathrm{C})$ gives the following result.

PROPOSITION 7 For $F \subseteq F r_{\Omega}, \mathcal{R}_{F}$ satisfies $(H)$ and $(C)$ and hence if $\mathcal{V}$ is subtractive, is a radical class if and only if it is closed under extensions.

We next give a useful sufficiency condition on the set $F$ to ensure $\mathcal{R}_{F}$ is closed under extensions. Let $F r_{\Omega_{1}}$ be the free algebra in $\mathcal{V}$ on the generators $x, y_{1}, z_{1}, y_{2}, z_{2}, \ldots$. We view $F r_{\Omega}$ as a subset of $F r_{\Omega_{1}}$. Then $F \subseteq F r_{\Omega}$ is said to be $\mathcal{U}$-associating (or just associating when no confusion arises) if and only if for all $A \in \mathcal{U}$, whenever there are $g, h \in F, r, a_{i} \in A$ and $b_{j} \in A$ for which $g\left(h\left(r, a_{i}\right), b_{j}\right)=0$, there are also $f \in F$ and $c_{k} \in A$ such that $f\left(r, c_{k}\right)=0$. (Note that $a_{i}$ here refers to $n-1$ elements of $A$ where $n$ is the number of generators involved in $h$, etc.) This notion is defined in greater generality but restricted to multioperator groups in [14].

We say $F \subseteq F r_{\Omega}$ as above is strongly associating if for all $f, g \in F$ there exist $h \in F$ and $g_{i} \in F r_{\Omega_{1}}$ such that

$$
f\left(g\left(x, y_{i}\right), z_{i}\right)=h\left(x, g_{i}\left(x, y_{j}, z_{j}\right)\right)
$$

It is easy to establish that if $F$ is strongly associating, then it is associating.

Two special cases arise frequently. If $f$ lies in the free algebra on one generator only, the associating condition for $\{f\}$ asserts that for all $A \in \mathcal{U}$, if $f(f(a))=0$ for some $a \in A$ then $f(a)=0$ also; $\{f\}$ is strongly associating if and only if $f(f(x))=f(x)$. If $F=\{f\}$ in the free algebra on two generators $x, y$, the associating condition says that for any $A \in \mathcal{U}$, if $f(f(a, b), c)=0$ for any $a, b, c \in A$, then there exists $d \in A$ for which $f(a, d)=0$; the strongly associating condition says that there is $g(x, y, z)$ for which $f(f(x, y), z)=f(x, g(x, y, z))$.

THEOREM 10 For $F \subseteq F r_{\Omega}, \mathcal{R}_{F}$ satisfies (E) in $\mathcal{U}$ if $F$ is associating.

PROOF. Suppose $A / N, N \in \mathcal{R}_{F}, N$ normal in $A \in \mathcal{U}$. Suppose $r \in A$. Then there exist $a_{i} \in A$ and $f \in F$ for which $f\left(r, a_{i}\right) \rho_{N} 0$, that is, $f\left(r, a_{i}\right) \in N$, so there exists $g \in F$ for which $g\left(f\left(r, a_{i}\right), b_{j}\right)=0$. Hence there exists $h \in F$ for which $h\left(r, c_{k}\right)=0$. Hence $A \in \mathcal{R}_{F}$ and (E) is established.

From Proposition 7, we obtain the following.

COROLLARY 4 Let $\mathcal{U}$ be a universal class in $\mathcal{V}, F \subseteq F r_{\Omega}$ associating. Then $\mathcal{R}_{F}$ satisfies $(H)$, $(E)$ and $(C)$, and so if $\mathcal{V}$ is subtractive, $\mathcal{R}_{F}$ is a radical class.

The three ring examples have $F$ strongly associating, but the radical class of Boolean rings, which is $\mathcal{R}_{\{f\}}$ where $f(x)=x^{2}-x$, does not (and indeed $\{f\}$ is not even associating).

A wide variety of examples arises in the following way. Suppose $\mathcal{V}$ is a 0 -normal variety with $*$ a (possibly derived) binary associative operation in the variety. Let $f(x, y)=x * y$ and $F=\{f\}$. 
Trivially $F$ is strongly associating, with $g(x, y, z)=f(y, z)$ in $f(f(x, y), z)=f(x, g(x, y, z))$. By analogy with the Jacobson radical of ring theory we call this class $\mathcal{J}_{\mathcal{V}, *}$ in general, or simply $\mathcal{J}_{*}$ if the context is clear. Note that $\mathcal{J}_{*}$ consists of those algebras in $\mathcal{V}$ for which $*$ is a group operation only if 0 is an identity for $*$ (in the sense that $0 * x=x * 0=x$ holds in the variety).

With $\mathcal{V}$ and $*$ as above, one can also define $f(x, y, z)=y * x * z$, again inducing a strongly associating $\{f\}$ since $f(f(x, y, z), u, v)=u * y * x * z * v=f(x, u * y, z * v)$, and we define $\mathcal{K}_{\mathcal{V}, *}=\mathcal{R}_{\{f\}}$; again we write $\mathcal{K}_{*}$ if the context is clear. Evidently $\mathcal{J}_{*} \subseteq \mathcal{K}_{*}$. If 0 is an identity for $*$, the condition of membership of this class is that the monoid under $*$ is simple, meaning there are no proper ideals of the associated semigroup. In ring theory, the class $\mathcal{K}$ of all rings $R$ for which $(R, \circ, 0)$ is a simple monoid was considered in [15], where it was shown to properly contain $\mathcal{J}$.

\section{Examples}

\section{EXAMPLE 1 Hoops}

The variety of hoops (see for example [2] for an excellent introduction) provides our most satisfying examples, with some of the most fundamental classes of hoops turning out to be radical. Some of the key results used in this section and referred to in [2] originally appeared in [3] and [4] in a more general setting.

Hoops are commutative monoids equipped with an additional binary operation $\rightarrow$ satisfying the three identities:

1. $x \rightarrow(y \rightarrow z)=(x y) \rightarrow z$,

2. $x \rightarrow x=1$ and

3. $(x \rightarrow y) x=(y \rightarrow x) y$.

There is a natural partial order on a hoop given by $x \leq y$ when $x \rightarrow y=1$. Indeed hoops can be characterised as ordered commutative monoids $S$ with the property that $x \leq y$ if and only if $x=y z$ for some $z \in S$, and for which $x \rightarrow y=\max \{z \in S \mid x z \leq y\}$ exists for all $x, y \in S$. It is readily shown that 1 is the largest element under the ordering. The ideals of a Dedekind domain $R$, under ideal product, with the partial order being inclusion, provide an example: $I \rightarrow J=\{x \in R \mid x I \subseteq J\}$. Every Heyting algebra is a hoop in which $x^{2}=x$, if one neglects the join. Another example is the free monoid on one generator $\langle a\rangle$, with $a^{n} \rightarrow a^{m}=a^{\max (m-n, 0)}$; this hoop is called $C_{\infty}$ in [2].

Hoops form a 0-normal variety: $\{1\}$ is a subhoop of any hoop, and letting $d(x, y)=(x \rightarrow$ $y)(y \rightarrow x)$, it is easily seen that $d(x, y)=1$ if and only if $x=y$. The variety is even Malcev and hence subtractive; see [2]. The normals are exactly filters: $N$ is a filter if $1 \in N, N$ is closed under multiplication, and $b \geq a \in N$ implies $b \in N$. Because filters are transitive in hoops 
$(F \triangleleft G \triangleleft H \Rightarrow F \triangleleft H)$, it is immediate that the universal class of hoops is normal in the sense of [16], and so all of the consequences of this important property follow.

Two useful simple facts in what follows are: (i) for any two elements $a, b$ of a hoop, $a b=b$ if and only if $a \rightarrow a b=1$; and (ii) $a b \rightarrow b=1$ for all $a, b$ in a hoop.

Let $k$ be a positive integer. In [2], a hoop is called $k$-potent if it satisfies the identity $x^{k+1}=x^{k}$.

PROPOSITION 8 The variety of $k$-potent hoops is an SSR class.

PROOF. Let $f(x)=x^{k} \rightarrow x^{k+1}$ in the free hoop on a single generator. Obviously, $\mathcal{R}_{\{f\}}$ is the class of $k$-potent hoops.

Now if $f(f(a))=1$ for any $a \in H$, a hoop, letting $b=f(a)=a^{k} \rightarrow a^{k+1}$, this says that $b^{k}=b^{k+1}$. But $b a^{k} \leq a^{k+1}$, with $b$ maximal with this property, so $b \geq a$, and so $a=b w$ for some $w \in H$, but also $b a^{k}=a^{k+1}$ (since $a a^{k}=a^{k+1}$ and $b \geq a$ ). Hence $a^{k+1}=b(b w)^{k}=b^{k+1} w^{k}=$ $b^{k} w^{k}=(b w)^{k}=a^{k}$, that is, $f(a)=1$. Hence $\{f\}$ is associating and so satisfies (E) by Corollary 4. Since the $k$-potent hoops form a variety and filters are transitive in hoops, they have attainable identities and hence form an SSR class by Corollary 3.

The implicative semilattices (subreducts of Heyting algebras to $(\wedge, \rightarrow, 1)$ ) are exactly the 1 potent hoops. By comparison, the analogous $F=\left\{x^{2}-x\right\}$ for rings is not associating, though the class of Boolean rings satisfies (E) and so is an SSR class. Note that $C_{\infty}$ is evidently not $k$-potent for any $k$, and indeed has no $k$-potent filters for any $k$, and so is semisimple with respect to every $k$-potent radical.

Define the class $\mathcal{M}$ of hoops to consist of those hoops $S$ such that for all $x \in S$ there exists $y \in S$ such that $x y=y$. If a hoop is $k$-potent for some $k$, then it is in $\mathcal{M}$. On the other hand, $C_{\infty}$ is obviously not in $\mathcal{M}$.

PROPOSITION $9 \mathcal{M}$ is a radical class.

PROOF. Letting $f=y \rightarrow x y$, it is clear that $\mathcal{M}=\mathcal{R}_{\{f\}}$. Suppose $a, b, c \in H$, a hoop, satisfy $f(f(a, b), c)=1$, that is, $(b \rightarrow a b) c=c$. Then $b c=b(b \rightarrow a b) c=a b(a b \rightarrow b) c=a b c$, so $(b c) \rightarrow a(b c)=1$, or $f(a, b c)=1$, so $\{f\}$ is associating.

It is not hard to see that $C_{\infty}$ has no regular filters and so is semisimple: $\mathcal{M}\left(C_{\infty}\right)=0$. Note that $\{f\}$ above is not strongly associating, as easy examples show.

Because hoops have transitive normals, by Theorem 9 , the anti- $k$-potent hoops for some $k$ (those for which the implication $x^{k+1}=x^{k} \Rightarrow x=1$ holds), constitute a semisimple quasivariety of hoops. An example is $C_{\infty}$. Obviously any such hoop is semisimple relative to the $k$-potent radical, but the converse fails in general. For instance, the hoop $\{1, a, b\}$, with $a^{n}=b$ for all $n>1$ and $1>a>b$, is not 1 -potent-semisimple but $b^{2}=b$.

EXAMPLE 2 Simploids 
Now obviously hoops are never groups (unless they are $\{1\}$ ), so $\mathcal{J}_{\times}=\mathcal{K}_{\times}$is trivial (where $\times$ is the monoid product). Next we consider a variety where the opposite behaviour occurs.

We say the algebra $A$ equipped with monoid structure $(A, \times, 1)$, with $\times$ usually denoted by juxtaposition, and two additional binary operations $\uparrow$ and $\downarrow$, is a simploid if it satisfies the following identities:

1. $x \uparrow x=x \downarrow x=1$; and

2. $(x \uparrow y) x(x \downarrow y)=y$.

Obviously every simploid is simple as a semigroup; hence the name given to these algebras. The variety of simploids is 0-normal: $\{1\}$ is a subalgebra, and $x \uparrow y=x \downarrow y=1$ if and only if $x=y$. Moreover, the variety has permuting congruences, as is shown by the existence of the term $\rho(x, y, z)=(y \uparrow x)(y \uparrow z) y(y \downarrow z)(y \downarrow x)$; then $\rho(x, x, z)=z$, and $\rho(x, z, z)=x$. Hence it is certainly subtractive and so both $\mathcal{J}_{\times}$and $\mathcal{K}_{\times}$are radical classes. Indeed $\mathcal{K}_{\times}$is the class of all simploids since the monoid part of a simploid is simple as a semigroup.

Any group $G$ is a simploid if one defines $x \uparrow y=y x^{-1}$ and $x \downarrow y=1$, so $\mathcal{J}_{\times}$is non-trivial. For an example which is not in $\mathcal{J}_{\times}$, consider the bicyclic semigroup $S$ on generators $a, b$, which is the monoid freely generated by $a, b$ subject to the relation $b a=1$, and consists of elements of the form $a^{n} b^{m}$ (where we define $a^{0}=b^{0}=1$ ), all of which are distinct. Products $\left(a^{n} b^{m}\right)\left(a^{r} b^{s}\right)$ are computed by using $b a=1$ as much as possible on the inner product $b^{m} a^{r}$. Define $\left(a^{n} b^{m}\right) \uparrow\left(a^{r} b^{s}\right)=a^{r-n}$ if $r \geq n$ and $b^{n-r}$ if $n>r$, and $\left(a^{n} b^{m}\right) \downarrow\left(a^{r} b^{s}\right)=b^{s-m}$ if $s \geq m$ and $a^{m-s}$ if $m>s$. Checking the simploid laws is routine, yet $S$ is not a group under multiplication. Hence $S \in \mathcal{K}_{\times} \backslash \mathcal{J}_{\times}$.

\section{EXAMPLE 3 EQ-monoids}

A commutative EQ-monoid $A$ is a commutative monoid having a distinguished submonoid $L_{A}$ which is a semilattice, such that $(a \bowtie b)=\max \left\{\alpha \in L_{A} \mid a \alpha=b \alpha\right\}$ exists for all $a, b \in A$. These are defined in greater generality in [17], where the monoid is not required to be commutative. They are also considered in [6], where additional operations are also possible.

It is possible to characterise commutative EQ-monoids equationally as follows: they are commutative monoids equipped with an additional binary operation $\bowtie$ such that

1. $(a \bowtie a)=1$ (reflexive rule); and

2. $f(a)(a \bowtie b)=f(b)(a \bowtie b)$ for each derived unary EQ-monoid operation $f$ (the replacement rule).

The replacement rule is in fact an axiom scheme. It is the basis of algebraic reasoning inside commutative EQ-monoids. For example, it lets us show that $a(a \bowtie b)=b(a \bowtie b)$, obtained by setting $f(x)=x$ in the replacement rule (this is immediate from the definition of an EQ-monoid anyway). Likewise, the law $(a \bowtie b)(d b \bowtie c)=(a \bowtie b)(d a \bowtie c)$ follows on letting $f(x)=(d x \bowtie c)$, 
and so on. We use these and various other easy corollaries of the replacement rule freely in what follows.

We should give some examples. If $\left(B, \vee, \wedge, 0,1,^{\prime}\right)$ is a Boolean algebra, then $(B, \wedge, 1)$ is a monoid, and is an EQ-monoid if $L_{B}=B$; in this case $(a \bowtie b)$ is $a \leftrightarrow b=(a \wedge b) \vee\left(a^{\prime} \wedge b^{\prime}\right)$, the "if-and-only-if" connective, as is easily checked. If $M$ is a monoid with zero element 0 , then it is an EQ-monoid if one lets $L_{M}=\{0,1\}$ : then $(a \bowtie a)=1$ for all $a \in M$, and $(a \bowtie b)=0$ for all unequal $a, b \in M$. Let $\left\{M_{x} \mid x \in X\right\}$ be a family of such monoids, with $M=\Pi_{x \in X} M_{x}$ the direct product of the $M_{x}$. Then $M$ is an EQ-monoid if $L_{M}$ consists of the elements of $M$ whose entries are 1 on a fixed subset of $X$ and 0 elsewhere. This kind of example can be characterised equationally (at least if 0 is admitted as an additional nullary operation); see [17].

Letting $d(x, y)=(x \bowtie y)$ easily shows the variety of EQ-monoids is 0-normal since $(x \bowtie x)=1$ and $x(x \bowtie y)=y(x \bowtie y)$.

We now summarise some of the most important properties of EQ-monoids. Each of these facts is shown in [17] (Proposition 1.9, Theorem 2.2 and Proposition 2.6).

THEOREM 11 Let $A$ be an EQ-monoid.

1. The operation $\wedge$ given by $a \wedge b=a(a \bowtie b)$ for all $a, b \in A$ is a semilattice operation extending that on $L_{A}$, with $a \leq b$ (the semilattice ordering) if and only if $a=b \alpha$ for some $\alpha \in L_{A}$.

2. a normal $N$ is a filter of $(A, \wedge$ ) containing 1 (and we call $N$ a normal filter of $A$ in this case).

3. If $p$ is an additional $n$-ary operation defined on $A$ and satisfies

$$
p\left(a_{1}, a_{2}, \ldots, a_{n}\right) \alpha=p\left(a_{1}, a_{2}, \ldots, a_{j} \alpha, \ldots, a_{n}\right) \alpha
$$

for all $a_{i} \in A$, all $j$, and all $\alpha \in L_{A}$, then all EQ-monoid congruences on $A$ also respect $p$.

It follows easily that principal normal filters can be written as $\left\langle\alpha>=\{a \mid a \geq \alpha\}, \alpha \in L_{A}\right.$, and that normals are transitive in this variety. (If $a \in N$, a normal filter, then so is $(a \bowtie 1)=a \wedge 1$.)

The condition on $p$ in the third part above says that $p$ is regular as defined in [6], which is the case if and only if the replacement rule extends to expressions involving $p$ also, as is shown in [6]. Both the monoid product and $\bowtie$ satisfy this condition in the current commutative setting.

The isomorphism theorem $(I \vee J) / J \cong I /(I \cap J)$ is not satisfied in the variety of commutative EQ-monoids, as is shown in [17]. Hence from Corollary 1, and despite the simple internal description of normals similar to that for hoops, the variety of commutative EQ-monoids is not subtractive.

Still, normal filters are transitive in EQ-monoids, so Theorem 9 applies. For any $n$, the semisimple quasivariety given by $x^{n}=1 \Rightarrow x=1$ is non-trivial, containing all EQ-monoids which are semilattices (and there are many such - see [6]). However it will not contain the example $\{0, a, 1\}$, 0,1 a zero and an identity respectively, with $a^{2}=1$ and $(x \bowtie y)=1$ if $x=y$ and 0 otherwise. Note 
that this is a non-trivial semisimple class in a non-subtractive universal class. Many interesting semisimple quasivarieties arise in this way, for example that consisting of all EQ-monoids with no non-trivial left or right invertible elements (given by the implication $x y=1 \Rightarrow x=y=1$ ). In all cases the radical is the smallest filter $F$ for which $f\left(a_{i}\right) \in F$ implies each $a_{i} \in F$, as in the proof of Theorem 9 .

Let $\mathcal{V}$ be the variety of EQ-monoids $A$ equipped with an additional associative and commutative binary operation $\vee$ satisfying $x(\alpha \vee \beta)=x \alpha \vee x \beta$ and $\alpha \vee 1=1$ (where $x \in A$ and $\alpha, \beta \in L_{A}$ ), the variety of distributive EQ-monoids. (These were first defined in [6], where the definition allowed $\checkmark$ as well as $\times$ to be non-commutative, unnecessary here.) This variety is Malcev (as is shown in [6]) by consideration of the term $\rho(x, y, z)=x(y \bowtie z) \vee z(x \bowtie y)$, and hence is subtractive. Note that any Heyting algebra is a distributive EQ-monoid in which $(a \bowtie b)=(a \rightarrow b)(b \rightarrow a)$, the monoid product is meet and $\vee$ is join, with 1 the top element. Moreover any Heyting algebra $H$ is in $\mathcal{J}_{\vee}$, since $a \vee 1=1$ for all $a \in H$. (If we also require $(a \vee b) \alpha=a \alpha \vee b \alpha$ for all $a, b \in A$ and $\alpha \in L_{A}$ - clearly satisfied by the Heyting algebra examples - then $\vee$ is regular, and so congruences of the underlying EQ-monoid also respect $\vee$ by Theorem 11, so normals are still filters containing 1, which satisfy the transitivity property, and so the results for normal classes in [16] apply.)

To show $\mathcal{J}_{\vee}$ is not all distributive EQ-monoids, consider the EQ-monoid of natural numbers (including zero) $N$ under multiplication, with $(m \bowtie n)=1$ if $m=n$ and 0 if $m \neq n$. Define $m \vee n=\max (m, n)$, making $N$ a distributive EQ-monoid, in which $L_{N}=\{0,1\}$, the two-element distributive lattice under $\vee$ and $\cdot$. Obviously this example is not in $\mathcal{J}_{\vee}$; indeed because $N$ is simple (its only filter containing 1 is itself), it is semisimple. (Note that $\vee$ satisfies $(a \vee b) \alpha=a \alpha \vee b \alpha$ in this example.)

In ring theory it is occasionally possible to define the radical of a ring to be all elements satisfying some particular property. For example, for commutative (associative) rings, the nil radical of $R$ is $\mathcal{N}(R)=\left\{a \in R \mid a^{n}=0\right.$ for some $\left.n>0\right\}$, and then $R$ is nil-semisimple if and only if it satisfies each of the implications $x^{n}=0 \Rightarrow x=0, n>0$. We now give an example of this form in a variety of EQ-monoids with an additional unary operation $I$. All of the ideas of this section are brought together in this example.

We say $A$ is an interior EQ-monoid if it possesses a unary operation $I$ for which $I(I(a))=I(a)$, $I(1)=1, I(a \wedge b)=I(a) \wedge I(b)$ and $I(a) \alpha=I(a \alpha) \alpha$, for all $a, b \in A$ and $\alpha \in L_{A}$. The last identity is simply regularity of $I$, so normal filters of the EQ-monoid induce congruences which $I$ also respects, by Theorem 11: $a \rho b$ implies $I(a) \rho I(b)$.

This class of interior EQ-monoids contains all EQ-monoids: define $I(a)=1$ for all $a$; hence it is not subtractive. On the other hand, let $I(a)=a$ in any EQ-monoid, giving an interior EQ-monoid in which $I(a)=1$ only if $a=1$.

Note that $f(x)=I(x)$ is (strongly) associating, so the equation $I(x)=1$ defines a variety which satisfies (E), and restricting to a subtractive subvariety or enrichment (for instance Heyting 
semilattices given by $x=(x \bowtie 1)$, or distributive EQ-monoids), it would be a radical class simply by Corollary 4 . Nonetheless we have the following result.

THEOREM 12 The variety $\mathcal{R}$ of all interior EQ-monoids satisfying $I(x)=1$ is a radical class with $\mathcal{R}(A)=\{a \in A \mid I(a)=1\}$ for any $A$. The corresponding semisimple class is the quasivariety satisfying $I(x)=1 \Rightarrow x=1$.

PROOF. Because of transitive normals, the quasivariety of interior EQ-monoids satisfying $I(x)=$ $1 \Rightarrow x=1$ is a semisimple class by Theorem 9 . From the proof of Theorem 2 , the radical of $A$ is the smallest normal filter which when factored out gives something in this quasivariety. In any interior EQ-monoid $A$, this obviously includes the set $\mathcal{I}(A)=\{a \in A \mid I(a)=1\}$. However, noting that $1 \in \mathcal{I}(A), \mathcal{I}(A)$ is a normal filter of $A$, and that $A / \mathcal{I}(A)$ has no such elements $b$ for which $I(b)=1$ - if it did then $A$ would have an element $c \notin \mathcal{I}(A)$ for which $I(c) \in \mathcal{I}(A)$, so $I(c)=I(I(c))=1$, a contradiction. Hence $\mathcal{R}(A)=\mathcal{I}(A)$, and obviously the radical class is the variety satisfying $I(x)=1$.

Defining $I(a)=a$ for all $a$ in any EQ-monoid gives a semisimple example, while defining $I(a)=1$ for all $a$ gives a radical example.

\section{EXAMPLE 4 Loops}

We close with a non-associative example. In [8], Gardner briefly alludes to the possibility of doing radical theory at the level of loop theory, but decides to draw the line of algebraic generality at (multi-operator) groups. Recall that a loop is an algebra with three binary operations $\cdot, \backslash$ and / and one nullary 1 satisfying

1. $x \backslash(x \cdot y)=y,(x \cdot y) / y=x$;

2. $x \cdot(x \backslash y)=y,(x / y) \cdot y=x$;

3. $x \cdot 1=1 \cdot x=x$.

Thus an associative loop is a group. The variety of loops is 0 -normal since $\{1\}$ is a subloop of any loop and $d(x, y)=x / y$ is an appropriate term for 0-regularity. It is Malcev also, hence subtractive.

Let $f(x)$ be an element of the free loop on one generator $x$. Define the class $\mathcal{T}_{f}$ to be all loops $A$ such that for all $a \in A, f^{n}(a)=1$ for some $n>0$. Then $f^{i}\left(f^{j}(x)\right)=f^{i+j}(x)$, so $F=\left\{f_{i} \mid i=1,2, \ldots\right\}$ is associating and so $T_{f}=\mathcal{R}_{F}$ is a radical class by Corollary 4 . Note that $f(x)=x^{m}$ for squarefree $m$ reduces for abelian groups to those groups for which every element has order a product of primes dividing $m$, so these $f$ provide non-trivial loop radicals too.

Similarly, the divisible radical for abelian groups can be generalised as follows. Let $f$ be as before and let $\mathcal{D}$ be the class of all loops $A$ for which, for all $x \in A$ there is $n>0$ and $y \in A$ for which $x=f^{n}(y)$. Then $F=\left\{f^{n} \mid n>0\right\}$ is again associating and so $\mathcal{R}_{F}$ is a radical class. Letting $f(x)=x^{m}$ as above gives various divisible radicals. 


\section{Concluding remarks}

Our main motivation for this article was the hope that much of Kurosh-Amitsur radical theory for rings (and more generally multi-operator groups) would make sense for 0-regular varieties in which all operations preserve 0. To some extent based on the work of Gardner on radicals of idempotent algebras [9], we developed a theory of radicals in this context. As in [9], the theory worked moderately well in full generality (although the usual homomorphic closure axiom needed to be altered), but worked better if the isomorphism theorem $(I \vee J) / J \cong I /(I \cap J)$ was assumed to hold.

Soon after completing this work on radicals in 0-regular varieties, we discovered that Puczyłowski [16] had already defined a lattice-based notion of universal class in which Kurosh-Amitsur radicals could be defined. The special case of subtractive 0-regular varieties in which all operations preserve 0 provided examples of his universal classes, and our definition of a radical class coincided with his in this case. It became clear that much of the work on 0-regular varieties generalised to a setting similar to Puczyłowski's but a little more general; this corresponded to moving from subtractive to general universal classes (in some ambient 0-normal variety).

Since then, thanks to the comments of the anonymous referee, we became aware that a farreaching generalisation of radical theory, applying in general categories, had already been developed in the work of Márki, Mlitz and Wiegandt, [13]. In particular, the desired generalisation of Puczyłowski's lattice approach proved to be a special case. So our original arguments in that setting could be eliminated, with the corresponding facts simply stated as special cases of results holding in [13].

In the present setting, it remains a task of further work to discover more non-subtractive examples, notably non-quasivariety examples. There is also interest in resolving some other issues, such as whether (R1) is implied by (H) in the presence of (R2) and (R3); in the idempotent algebras case considered by Gardner in [9], this question is resolved in the negative, and the cost of using (H) instead of (R1) is, among other things, that "semisimple classes" need no longer be subdirectly closed.

Deciding when semisimple classes admit a simpler characterisation, along the lines of the familiar one for associative rings, would also be of interest. Finally, the non-radical theoretic question as to whether the isomorphism theorem $(I \vee J) / J \cong I /(I \cap J)$ holding in a 0-normal variety is equivalent to it being subtractive should be resolvable.

\section{Acknowledgements}

We would like to thank the referee for pointing us in the direction of [13], as well as for a number of other useful comments and minor corrections. The referee's careful consideration of this article has led to its significant improvement from the original submitted version. 


\section{References}

[1] P. Agliano and A. Ursini, Ideals and other generalizations of congruence classes, J. Austral. Math. Soc. (Ser. A), 53 (1992), 103-115.

[2] W. J. Blok and I. M. A. Ferreirim, On the structure of hoops, Alg. Univ., 43 (2000), 233-257.

[3] B. Bosbach, Komplementäre Halbgruppen. Axiomatik und Arithmetik, Fund. Math., 64 (1969), 257-287.

[4] B. Bosbach, Komplementäre Halbgruppen. Kongruenzen und Quotienten, Fund. Math., 69 (1970), 1-14.

[5] P. M. Cohn, Universal Algebra, Harper and Row, London (1965).

[6] D. Fearnley-Sander and T. Stokes, Varieties of equality structures, Int. J. Alg. Comp., 13 (2003), 463-480.

[7] B. J. Gardner, Radicals and varieties, in Colloquia Mathematica Societatis János Bolyai 38, Radical Theory, Eger, Hungary, 1982, 93-133 (1985).

[8] B. J. Gardner, Radical Theory, Longman Scientific and Technical, Harlow, Essex, UK (1989).

[9] B. J. Gardner, Radical decompositions of idempotent algebras, J. Austral. Math. Soc. (Ser. A), 36 (1984), 213-236.

[10] H. P. Gumm and A. Ursini, Ideals in universal algebras, Alg. Univ., 19 (1984), 45-54.

[11] H.-J. Hoehnke, Radikale in allgemeinen Algebren, Math. Nachr., 32 (1966), 347-383.

[12] S. R. Kogalovskii, Structural characteristics of universal classes, Sibirsk. Mat. Z., 4 (1963), 97-119 (in Russian).

[13] L. Márki, R. Mlitz and R. Wiegandt, A General Kurosh-Amitsur Radical Theory, Comm. Algebra, 16 (1988), 249-305.

[14] N.R. McConnell and T. Stokes, Equationally Defined Radical Classes, Bull. Aust. Math. Soc., 47 (1993), 217-220.

[15] N.R. McConnell and T. Stokes, Rings having simple adjoint semigroup, in Proceedings of AGRAM2000 (Abelian Groups, Rings and Modules conference, July 2000, Perth, Australia), AMS Contemporary Mathematics Series 273, American Mathematica Society, 203-208 (2001).

[16] E.R. Puczyłowski, On general theory of radicals, Alg. Univ., 30 (1993), 53-60.

[17] T. Stokes, On EQ-monoids, to appear in Acta Sci. Math.

[18] A. Ursini, On subtractive varieties, I, Alg. Univ., 31 (1994), 204-222. 
Department of Defence

Locked Bag 5076

Kingston

ACT 2604

Australia

Department of Mathematics

School of Computing and Mathematical Sciences

University of Waikato

Hamilton

New Zealand 